\section{Matrix metalloproteinase 9 polymorphism and outcome after myocardial infarction}

\author{
Sophie Rodius, ${ }^{1}$ Guillermo Mulliert, ${ }^{2}$ \\ Francisco Azuaje, ${ }^{1}$ Yvan Devaux, ${ }^{1}$ \\ Daniel R. Wagner ${ }^{1,3}$
}

\section{'Laboratory of Cardiovascular Research, Centre de Recherche Public-Santé, Luxembourg, Luxembourg; 'Laboratory of Crystallography, Magnetic Resonance and Modeling, UMR UHP-CNRS 7036,} Faculté des Sciences et Technologies, Nancy Université, Vandoeuvre-les-Nancy, France; ${ }^{3}$ Division of Cardiology, Centre Hospitalier, Luxembourg, Luxembourg

\section{Abstract}

Matrix metalloproteinase 9 (MMP9) is functionally implicated in the process of infarct healing. Several genetic variation of the MMP9 gene have been described, among which the MMP9 Arg668Gln polymorphism. In the present study, we assessed whether this polymorphism influences outcome after acute myocardial infarction (MI). One thousand forty-nine patients undergoing coronary angiography were genotyped for the MMP9 Arg668Gln polymorphism by TaqMan allelic discrimination assay. This population included 154 controls, 161 patients with non ST-elevation MI (NSTEMI), 504 patients with ST-elevation MI (STEMI), and 230 patients with angina. Frequency of the MMP9 Arg668Gln polymorphism in the global population was $25.1 \%$, and was comparable between all groups. STEMI patients had higher creatine phosphokinase (CPK), troponin T (TnT) and MMP9 plasma levels and had lower ejection fraction (EF) than NSTEMI patients. However, the polymorphism was not associated with infarct severity as determined by peak CPK and TnT levels, nor with $\mathrm{LV}$ remodeling and outcome as assessed by 1-month EF and NYHA class, as well as 2year mortality. In silico molecular modeling simulations predicted that the MMP9 polymorphism may decrease MMP9 activity, but this could not be verified by plasma determinations. This study investigated for the first time the association between the MMP9 Arg668Gln polymorphism and clinical outcome after acute MI. Our results indicate that the polymorphism does not seem to be associated with clinical outcome and in particular with the development of left ventricular dysfunction and heart failure.

\section{Introduction}

Left ventricular (LV) remodeling following myocardial infarction (MI), ultimately culminating in the development of heart failure (HF), is a complex phenomenon involving several biological processes such as the turnover of the extracellular matrix (ECM) and inflammation. Matrix metalloproteinases (MMP) degrade the myocardial ECM leading to deleterious remodeling and $\mathrm{LV}$ dysfunction. ${ }^{1}$ Among the different MMPs expressed in the heart and the circulation, the levels and activity of MMP9 are elevated after MI and correlate with LV dysfunction. ${ }^{2-7}$ Furthermore, we and others have identified MMP9 as a marker of LV remodeling and heart failure after MI. ${ }^{7-10}$ Interestingly, the prognostic value for LV remodeling of tissue inhibitor of MMP 1 (TIMP1), the main endogenous inhibitor of MMP9, has also been reported. ${ }^{8}$ These investigations identified MMP9 not only as a biomarker of HF but also as a key player in the remodeling process.

Several polymorphisms located both in the promoter or coding region of the MMP9 gene correlate with the occurrence and development of several forms of cancers. ${ }^{11-14}$ However, MMP9 polymorphisms have been poorly studied in the context of acute MI. ${ }^{15-17}$ One single nucleotide polymorphism (SNP, code rs17577) located in MMP9 coding sequence induces a change from a guanine to an adenine, resulting in the substitution of amino acid 668 from an arginine (Arg) to a glutamine (Gln). This polymorphism, called Arg668Gln, is within the hemopexin domain of MMP9 (PEX9) involved in homodimerization and binding to substrates and TIMP1. ${ }^{18}$ It is therefore conceivable that this polymorphism could modify the interaction between MMP9 and TIMP1, thereby altering MMP9 activity. Studies reported to date showed an absence of correlation between this SNP and MI. ${ }^{16,17}$ However, an association with cardiovascular risk factors and serum MMP9 levels was recently reported. ${ }^{19}$ The purpose of the present study was to determine whether the MMP9 Arg668Gln polymorphism influences outcome after acute MI.

\section{Materials and Methods}

\section{Patients}

The first group of patients included patients with acute MI who were enrolled in a national MI registry and treated with primary percutaneous coronary intervention. Acute ST-elevation MI was defined by the presence of chest pain $<12$ hours with significant ST elevation and peak creatine phosphokinase level above 600 units/L. Patients who had no ST-elevation
Correspondence: Daniel R. Wagner, Division of Cardiology, Centre Hospitalier Luxembourg, 4 rue Barblé, L1210 Luxembourg, Luxembourg. Tel: +352.26 .970 .300 - Fax: +352.26 .970 .396 . E-mail:wagner.daniel@chl.lu

Key words: matrix metalloproteinase 9, myocardial infarction, polymorphism, ventricular function.

Acknowledgments: the authors would like to thank Christelle Nicolas, Emilie Lieffrig, Bernadette Leners, Céline Jeanty, Malou Gloesener and Loredana Jacobs for expert technical assistance. The authors would also like to thank Claude Didierjean and Frédérique Favier for their contribution. This work was supported by grants from the National Fund for Research of Luxembourg, the Society for Research on Cardiovascular Diseases, and the Ministry of Culture, Higher Education and Research of Luxembourg. SR was recipient of a postdoctoral fellowship from the National Fund for Research of Luxembourg.

Contributions: YD, DRW, conceived the study; DRW, collected clinical samples. SR, generated genotypic data and participated in data analysis; YD, FA completed data analysis; GM, produced in silico modeling data. All authors participated in data interpretation and approved the manuscript final version.

Conflict of interest: the authors report no conflicts of interest.

Received for publication: 31 January 2011. Accepted for publication: 9 June 2011.

This work is licensed under a Creative Commons Attribution NonCommercial 3.0 License (CC BYNC 3.0).

(C) Copyright S. Rodius et al., 2011

Licensee PAGEPress, Italy

Cardiogenetics 2011; 1:e5

doi:10.4081/cardiogenetics.2011.e5

but positive cardiac markers were classified as non-STEMI (NSTEMI). All patients had successful mechanical reperfusion and stenting of the infarct artery within 12 hours of chest pain onset. Blood samples were obtained at the time of mechanical reperfusion. LV function was determined 1-month after MI with echocardiography. The second group of patients included patients with stable angina and unstable angina who underwent uncomplicated percutaneous coronary intervention (PCI). Cardiac enzymes were normal before and after the procedure. The third group of patients included normal controls with atypical chest pain, abnormal stress testing but normal coronary angiography. Clinical outcome was determined by the following end-points: ejection fraction (EF) measured 1-month after MI with echocardiography, New York Heart Association 
(NYHA) class evaluated at 1-month follow-up, and mortality at 2 years. The protocol has been performed conforming to the Helsinki Declaration and approved by the local ethics committee. Informed consent has been obtained from all subjects.

\section{Genotyping}

Genomic DNA was extracted from the buffy coat of centrifuged blood using FlexiGene kit 250 (Qiagen GmbH, Hilden, Germany) according to the manufacturer protocol. Detection of MMP9 polymorphism was performed by TaqMan SNP allelic discrimination on a BioRad iQ5 apparatus using the following primers and probes: $M M P 9$ forward primer: CTCAGCACCTGTCTCCTC, MMP9 reverse primer: GGGCATTTGTTTCCATTTCC, MMP9 wild-type probe: TAMRA-CCTCACCTCGGTACTGGA-BHQ2, MMP9 Arg668GIn probe: Texas Red-CCTCACCTTGGTACTGGAA-BHQ2. The PCR reaction was performed in a $20 \mu \mathrm{L}$ reaction mix containing $10 \mathrm{ng}$ of genomic DNA, $2 \mu \mathrm{L}$ of 10X AmpliTaq Gold buffer (Applied Biosystems, Life Technologies Corp., Carlsbad, CA, USA), $1.6 \mu \mathrm{L}$ of $25 \mathrm{mmol} / \mathrm{L} \mathrm{MgCl}_{2}$ (Applied Biosystems), $1.6 \mu \mathrm{L}$ of $2.5 \mathrm{mmol} / \mathrm{L}$ each $\mathrm{dNTPs}$ (Invitrogen Corp., san Diego, CA, USA), $0.8 \mu \mathrm{L}$ of each $10 \mu \mathrm{mol} / \mathrm{L}$ primer, $0.8 \mu \mathrm{L}$ of each 10 $\mu \mathrm{mol} / \mathrm{L}$ probe and $0.2 \mu \mathrm{L}$ of $5 \mathrm{U} / \mu \mathrm{L}$ AmpliTaq Gold (Applied Biosystems). The PCR reaction was composed of 1 cycle at $95^{\circ} \mathrm{C}$ for $3 \mathrm{~min}$, followed by 50 cycles at $95^{\circ} \mathrm{C}$ for $30 \mathrm{~s}, 60.5^{\circ} \mathrm{C}$ for 30 s and $72^{\circ} \mathrm{C}$ for $30 \mathrm{~s}$.

\section{Biochemical assays}

Plasma levels of MMP9 and TIMP1 were measured using ELISA from R\&D Systems Inc. (Minneapolis, MN, USA). Detection limits of the assays are $0.156 \mathrm{ng} / \mathrm{mL}$ for total MMP9 (cat \# DMP900), 0.005ng/mL for active MMP9 (cat\# FD9M00) and $0.08 \mathrm{ng} / \mathrm{mL}$ for TIMP1 (cat \# DTM100).

\section{Molecular modeling}

The model and energy analysis of the hemopexin domain of MMP9 (PEX9) was done using both monomers of pdb ID 1ITV. ${ }^{20}$ PEX9 Arg668Gln dimer was modeled using the same pdb ID. Model of PEX9/TIMP1 complex was done using chain A of pdb ID 1ITV and the inhibitor coordinates were taken from pdb ID 1UEA, chain B. ${ }^{21}$ The complex PEX9/TIMP1 was constructed using the protein-protein docking program MOLFIT [http://www.weizmann.ac iVChemical_Research_Support/molfit/home.h tml. ${ }^{22,23}$ ]. PEX9 (Arg 668Gln)-TIMP-1 complex was done from the docking result. All other molecular modeling studies were done using AMBER 8 using FF99 force field. ${ }^{24}$ Briefly hydrogens were added to pdb coordinates and minimized followed by solvatation of the models using an octahedron box. Energy of the sol- vated models was again minimized. Subsequently, the whole system was submitted to molecular dynamics, by means of an equilibration phase of $2.1 \mathrm{~ns}$ rising temperature from 0 $\mathrm{K}$ to $300 \mathrm{~K}$ followed by a production phase of $5.5 \mathrm{~ns}$ at $300 \mathrm{~K}$. Calculations of free energies was done with MM-PBSA using the production phase. In all complexes studied, chain A was used as receptor and chain $\mathrm{B}$ as ligand.

\section{Statistical analysis}

Comparisons between two groups of continuous data were performed with two-tailed ttest for data with a normal distribution and Mann-Whitney test for non-normally distributed data. Normality was determined using the Shapiro-Wilk test. Chi-square test with Yates correction for continuity was used for comparing frequencies. Comparisons between multiple groups were performed using one-way ANOVA. Kruskal-Wallis one-way ANOVA on ranks was executed for non-normally distribtests (Dunn's or Holm-Sidak method) were run to isolate the group or groups that differ from the others. SigmaPlot 11.0 software was used for these analyses.

\section{Results}

\section{Frequency of matrix metallopro- teinase 9 polymorphism}

The presence of MMP9 variation was determined by allelic discrimination using TaqMan assay. The frequency of the MMP9 Arg668Gln polymorphism was first determined in the entire population used in this study, comprising 1049 patients. Clinical characteristics can be found in Table 1.786 of the 1049 patients did not have the MMP9 variation (wt), 248 were heterozygous and only 15 were homozygous, i.e. $25.1 \%$ carried one or two mutated alleles (mut). The number of homozygous patients being very low, we analyzed together uted data. In case of significance, post-hoc

mutant homozygous and heterozygous patients.

We investigated whether the frequencies of MMP9 polymorphism differed between controls, i.e. patients with chest pain but normal coronary arteries $(n=154)$, patients with angina undergoing uncomplicated PCI $(n=230)$, and MI patients undergoing primary PCI subdivided into patients with ST-elevation (STEMI, $\mathrm{n}=504$ ) and patients without ST-elevation (NTEMI, $\mathrm{n}=161$ ). Clinical characteristics are gathered in Table 2. In all 4 groups, 20 to $28 \%$ of the patients carried the MMP9 variation. The frequencies of the polymorphism did not differ between the 4 groups (Figure 1).

\section{Association between matrix metal- loproteinase 9 polymorphism and infarct size}

To determine a potential relationship between the polymorphism and infarct severity, we compared peak CPK and TnT levels with the presence of the polymorphism among the 504 STEMI and the 161 NSTEMI patients. As expected, CPK and TnT plasma levels were significantly higher in STEMI than in NSTEMI patients. However, patients carrying or not the polymorphism had comparable CPK and TnT

Table 1. Characteristics of the entire population ( $\mathrm{n}=1049)$.

\begin{tabular}{lcc}
\hline Demographics & & \\
Age, y (median-range) & 63 & $22-91$ \\
Gender, male (n, \%) & 776 & 74 \\
\hline Body Mass Index (median-range) & 27 & $16-56$ \\
\hline Risk factors ( $\mathrm{n}, \%)$ & & \\
Diabetes & 228 & 21.7 \\
Hypertension & 517 & 49.3 \\
\hline Hypercholesterolemia & 475 & 45.3 \\
Tobacco & 425 & 40.5 \\
\hline Family history & 291 (580) & 50.2 \\
\hline When different from 1049, the number of available data is indicated \\
in parenthesis.
\end{tabular}

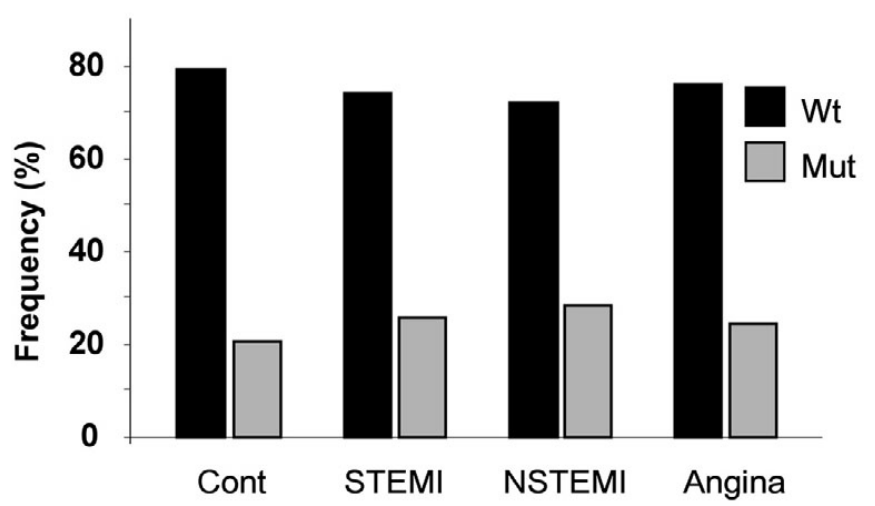
\begin{tabular}{l}
\hline Figure 1. \\
Frequency of the \\
MMP9 Arg668 \\
Gln polymor- \\
phism in con- \\
trols, STEMI, \\
NSTEMI and \\
angina patients. \\
Frequencies were \\
co m p r a b 1 e \\
between the 4 \\
groups $(\mathrm{P}=0.68)$. \\
Cont: controls.
\end{tabular} 
levels, both in the STEMI and in the NSTEMI groups (Figure 2A).

\section{Association between matrix metal- loproteinase 9 Arg668Gln poly- morphism and outcome after myocardial infarction}

LV EF was available for 468 of the 665 MI patients, NYHA class for 589 patients and 2year mortality for 603 patients (Table 2). The large majority of STEMI patients had a favorable outcome after MI with a median EF of $50 \%$ and a 2 -year mortality of $7.8 \% .91 .1 \%$ of the patients were in NYHA class of 1-2 and $8.9 \%$ in class of 3-4 (Table 2). Despite a lower EF in STEMI compared to NSTEMI patients (Table 2, $50 \%$ vs $55 \%$, respectively, $\mathrm{P}<0.001$ ), the presence of the MMP9 Arg668Gln variation was not associated with EF (Figure 2B). Similarly, 2year mortality was higher in the STEMI compared to the NSTEMI population (Table 2, $\mathrm{P}=0.04$ ), but it was not statistically significantly associated with the presence of the polymorphism (Figure 2A-B). Finally, no correlation between the genetic variation and NYHA class could be demonstrated (Figure 2A-B).

\section{Association between matrix metal- loproteinase 9 Arg668GIn} polymorphism and left ventricular remodeling after myocardial

\section{infarction}

Left ventricular remodeling was assessed by the EF measured by echocardiography at 1month follow-up. An EF > 40\% indicates the absence of remodeling and an $\mathrm{EF} \leq 40 \%$ indicates $\mathrm{LV}$ remodeling and dysfunction. To determine a potential correlation between the presence of the MMP9 polymorphism and LV remodeling after $\mathrm{MI}$, we divided the population of MI patients into two groups according to their EF ( $\leq 40 \%, n=79 ;>40 \%, n=389)$. See Table 3 for clinical characteristics. As expected, low EF patients were in a higher NYHA class $(\mathrm{P}=0.005)$ and had a higher 2-year mortality rate (18 vs. $5.4 \%, \mathrm{P}=0.002)$ than high $\mathrm{EF}$ patients (Table 3). Although the MMP9 variation appeared to be more frequent in high $\mathrm{EF}$ patients, this was not statistically significant (Figure 3).

Molecular modeling of the structure of mutated matrix metallopro-

\section{teinase 9}

In silico molecular modeling experiments were undertaken to study the impact of the Arg668Gln variation on MMP9 structure. Secreted as a pro-peptide, MMP9 and most particularly its hemopexin domain PEX9 can form dimers with both another MMP9 peptide or with TIMP1. Localization of the Arg668Gln
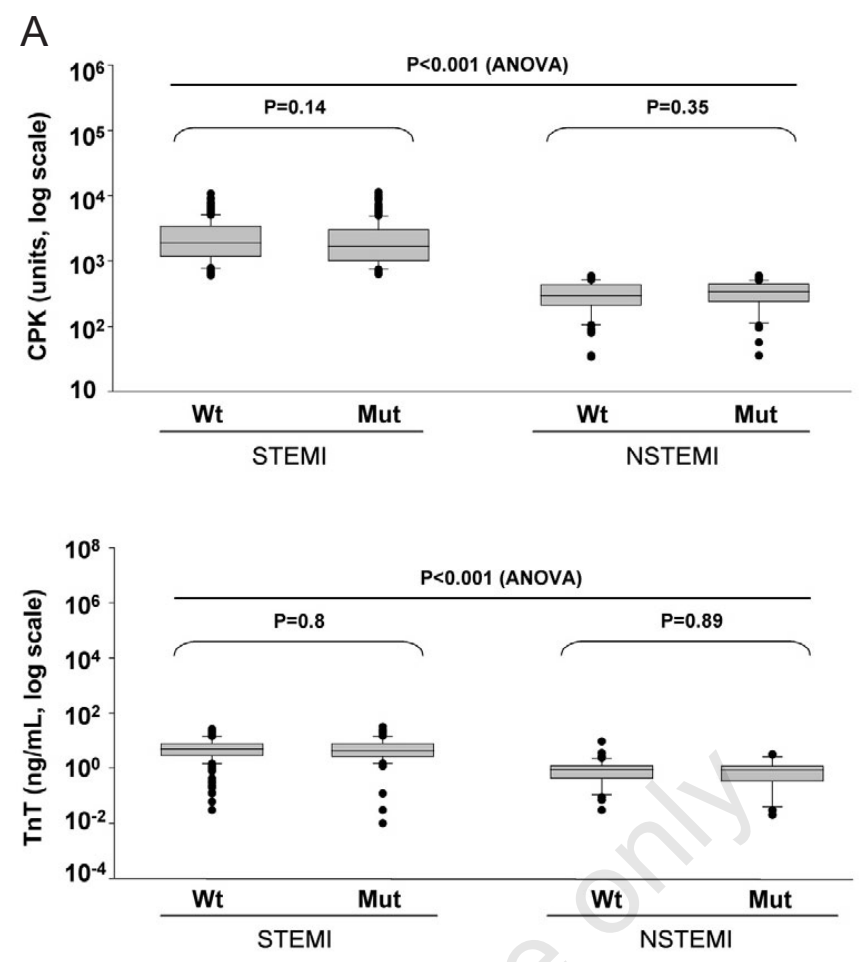

B
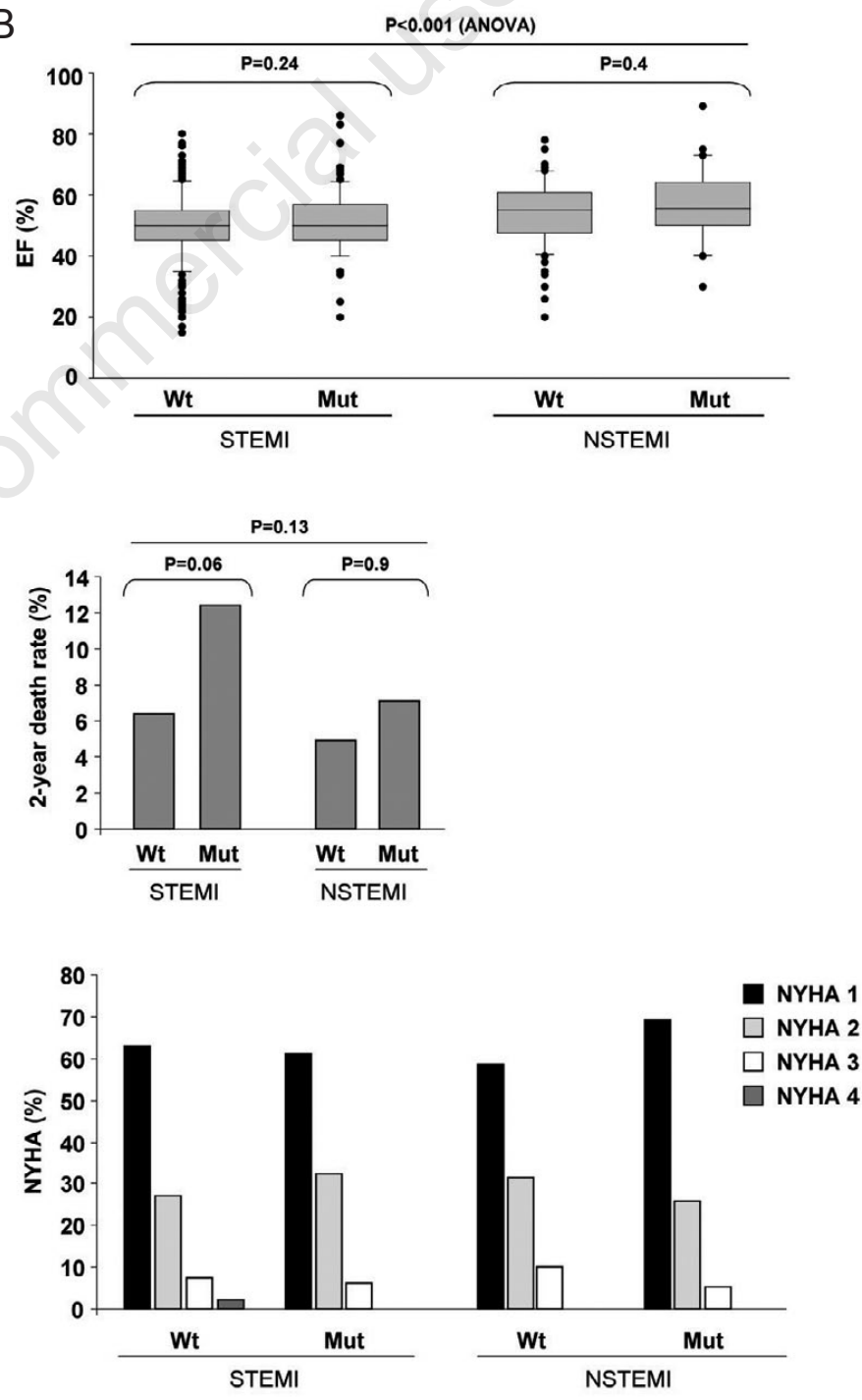

[Cardiogenetics 2011; 1:e5]
Figure 2. Association between MMP9 polymorphism, infarct size and clinical outcome after MI. (A) Infarct size was determined by peak CPK and TnT levels. (B) 1month EF, 2-year death rate and NYHA class were used as end-points representing clinical outcome after MI. STEMI patients had higher CPK and TnT levels and lower EF than NSTEMI patients. No statistically significant correlation was found between the presence of the Arg668Gln variation and infarct size or clinical outcome after MI. 
Table 2. Characteristics of controls, NSTEMI, STEMI and angina patients.

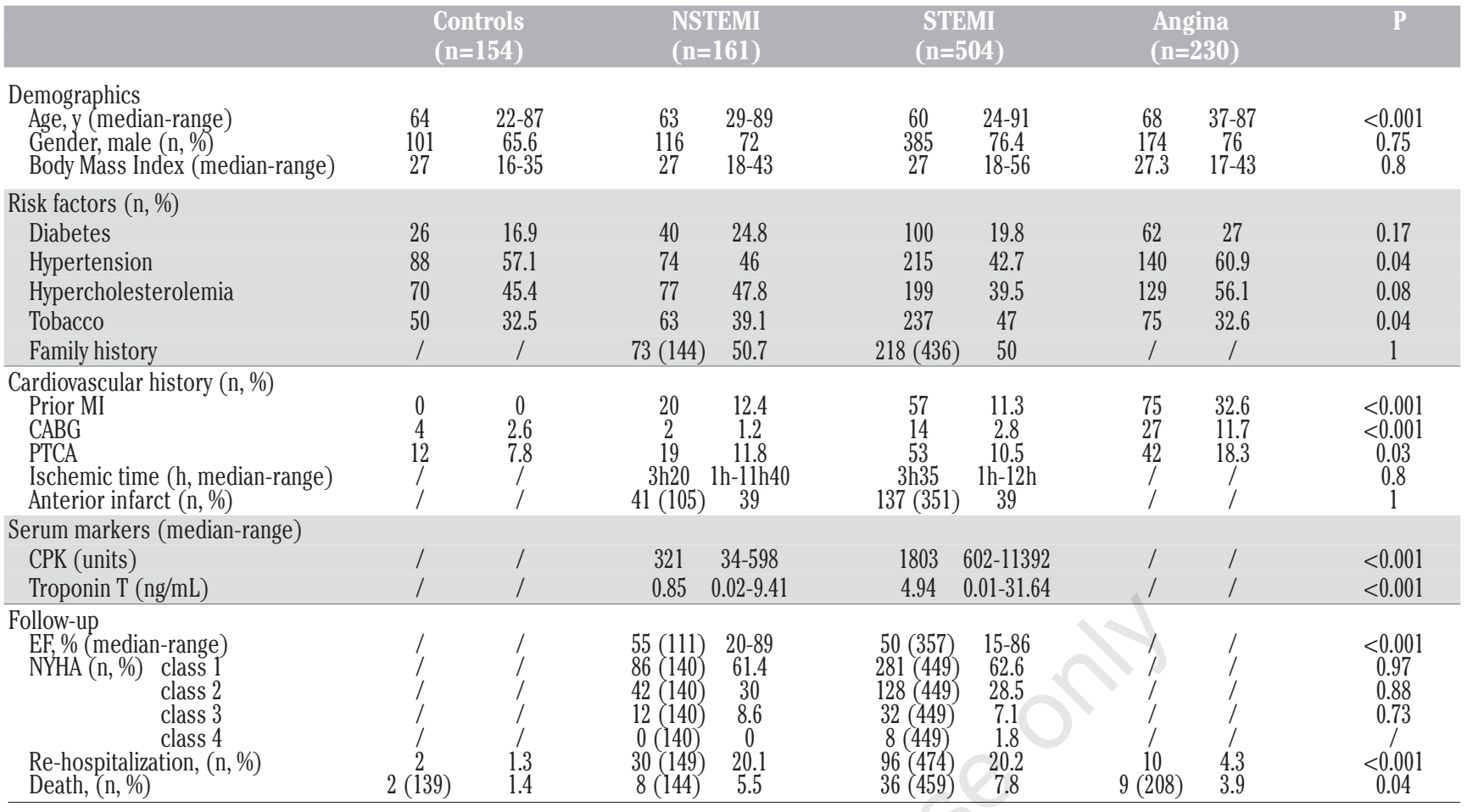

CABG, Coronary Artery Bypass Grafting; CPK, Creatine PhosphoKinase; EF, Ejection Fraction; MI, Myocardial Infarction; PTCA, Percutaneous Transluminal Coronary Angioplasty; NYHA, New York Heart Association class. When different from the total, available numbers of data are indicated in parenthesis.

polymorphism in the PEX9 domain suggested that it may modulate the formation of PEX9/PEX9 or PEX9/TIMP1 dimers. Both complexes are represented in Figure 4. Molecular dynamics data indicated that the Arg668Gln polymorphism is located $18 \AA$ away from the interface between the two PEX9 monomers, excluding a potential impact on the structure of PEX9/PEX9 dimer (Figure 4A). Additionally, the difference in binding energy between the wt PEX9/PEX9 dimer and the mutated PEX9/PEX9 dimer is less than 5\%, indicating similar stabilities of the two dimers.

Additional docking analyses between PEX9 and TIMP1 showed that the interaction interface of PEX9 would be different from the PEX9/PEX9 dimer. The residue 668 of PEX9 is located at a distance $<4 \AA$ from the contact zone between PEX9 and TIMP1, suggesting that it could establish direct interactions with TIMP1 (Figure 4B). Indeed, the presence of the genetic variation increases the number of hydrogen bounds from 8 (for the wt complex) to 18 for the mutated complex. This indicates that the variation stabilizes the PEX9/TIMP1 dimer. Consistently, binding energy of the mutated PEX9/TIMP1 dimer is near two-fold of wt PEX9/TIMP1 dimer. Therefore, this in silico approach suggested that the Arg668Gln polymorphism may favor the binding of MMP9 to TIMP1, thereby decreasing its activity.

Relationship between matrix met-
Table 3. Clinical characteristics of the 2 groups of MI patients: with LV dysfunction $(\mathrm{EF} \leq 40 \%)$ and without $\mathrm{LV}$ dysfunction $(\mathrm{EF}>40 \%)$.

$\mathrm{EF} \leq 40(n=79) \quad \mathrm{EF}>40(n=389) \quad \mathrm{P}$

\section{Demographics}

$\begin{array}{lccccc}\text { Age, y (median-range) } & 64 & 32-91 & 59 & 29-90 & 0.005 \\ \text { Gender, male (n, \%) } & 63 & 79.7 & 300 & 77.5 & 0.93 \\ \text { Body Mass Index (median-range) } & 26.6 & 20-38 & 27 & 18-47 & 0.08 \\ \begin{array}{l}\text { Risk factors (n, \%) } \\ \text { Diabetes }\end{array} & 22 & 27.8 & 76 & 19.6 & 0.24 \\ \text { Hypertension } & 34 & 43.6 & 157 & 40.7 & 0.86 \\ \text { Hypercholesterolemia } & 27 & 35.1 & 174 & 45.2 & 0.31 \\ \text { obacco } & 32 & 40.5 & 179 & 46 & 0.65 \\ \text { Family history } & 33(68) & 48.5 & 180(353) & 51 & 0.92\end{array}$

Cardiovascular history (n, \%)

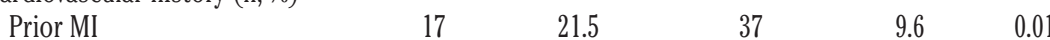

CABG

PTCA

$2 \quad 2.5$

Ischemic time (h, median-range) $\quad 3 \mathrm{~h} 40$

Anterior infarct (n, \%)

$29(52)$

$$
16.4
$$

1h10-9h45

10

10
37

$3 \mathrm{~h} 30$

2.6

0.01

$9.5-0.16$

Serum markers (median-range)

CPK (units)
Troponin T (ng/mL)

\section{Follow-up}

\begin{tabular}{|c|c|c|c|c|c|}
\hline EF, \% (median-range) & 35 & $15-40$ & 53 & $41-89$ & $<0.001$ \\
\hline NYHA (n, \%) class 1 & $31(65)$ & 47.7 & $222(356)$ & 62.3 & \\
\hline class 2 & $19(65)$ & 29.2 & $106(356)$ & 29.8 & \\
\hline class 3 & $13(65)$ & 20 & $23(356)$ & 6.5 & 0.0050 \\
\hline class 4 & $2(65)$ & 3.1 & $5(356)$ & 1.4 & \\
\hline Re-hospitalization, (n, \%) & 21 & 28.8 & 87 & 23.3 & 0.62 \\
\hline Death (n, \%) & $13(72)$ & 18 & $19(352)$ & 5.4 & 0.002 \\
\hline
\end{tabular}

CABG, Coronary Artery Bypass Grafting; CPK, Creatine PhosphoKinase; EF, Ejection Fraction; MI, Myocardial Infarction; PTCA, Percutaneous Transluminal Coronary Angioplasty; NYHA, New York Heart Association class. When different from the total, available numbers of data are indicated in parenthesis. 
alloproteinase 9 gene variation and plasma levels of matrix metalloproteinase 9 and TIMP-1

To address this assumption, we measured MMP9 and TIMP1 plasma levels in the population of STEMI and NSTEMI patients. Both active and total MMP9 levels were determined using specific assays. However, while all STEMI and NSTEMI patients were tested for total MMP9 and TIMP1, only 64 STEMI and 28 NSTEMI patients were tested for active MMP9. While total MMP9 level was slightly higher in the plasma of STEMI patients compared to NSTEMI patients, no significant association between the MMP9 variation and plasma levels of MMP9 or TIMP1 could be demonstrated (Figure 5). To rule out a potential bias due to blood sampling time after chest pain onset, we analyzed the levels of MMP9 and TIMP1 according to the ischemic time, i.e. the time between chest pain onset and blood harvesting (=reperfusion). Both MMP9 and TIMP1 levels were relatively stable during the 12 hours following acute MI, indicating that the measurements of these proteins was not influenced by the time of blood harvesting (Figure 6A-B).

\section{Discussion}

An association between plasma level of MMP9 and cardiovascular risk factors was recently reported, ${ }^{19}$ and MMP9 has already been identified as a marker of LV remodeling and heart failure after MI. ${ }^{7-10} \mathrm{~A}$ polymorphism has been discovered in MMP9 hemopexin domain PEX9: the MMP9 Arg668Gln variation, but to date no study was able to demonstrate a correlation between this polymorphism and MI. ${ }^{16,17}$ PEX9 being involved in MMP9 homodimerization and binding to MMP9 substrates and inhibitor, ${ }^{18}$ it was tempting to hypothesize that the genetic variation could alter MMP9 activity and the extent of matrix remodeling. However, in the present study, no significant association between the presence of the MMP9 Arg668Gln polymorphism and outcome after MI could be established.

We first analyzed the frequency of the MMP9 Arg668Gln polymorphism in 1049 patients undergoing coronary angiography. Our data showing a frequency of $25.1 \%$ are in accordance with the frequency reported in NCBI Reference SNP: $28.7 \pm 2.5 \%$ worldwide and $26.5 \pm 1.1 \%$ in the European population. Although only a limited number of patients without coronary artery disease or with stable angina were tested, the Arg668Gln variation did not appear to be more frequent in MI patients.

As expected, STEMI patients had lower EF than NSTEMI patients, higher peak CPK and
TnT levels, and a higher 2-year mortality. However, we found no significant association between the MMP9 polymorphism and peak CPK and TnT levels, 1-month EF and NYHA class, and 2-year mortality. These results indicate that the genetic variation is not associated with infarct severity and clinical outcome after MI. To investigate the relationship between the Arg668Gln variation and LV remodeling after MI, we divided the population of MI patients into a group of patients with preserved $\mathrm{LV}$ function (EF >40\%) and a group of patients with $\mathrm{LV}$ dysfunction $(\mathrm{EF} \leq 40 \%)$. As expected, clinical characteristics and outcome were significantly different between both groups. Patients with low EF were older, had

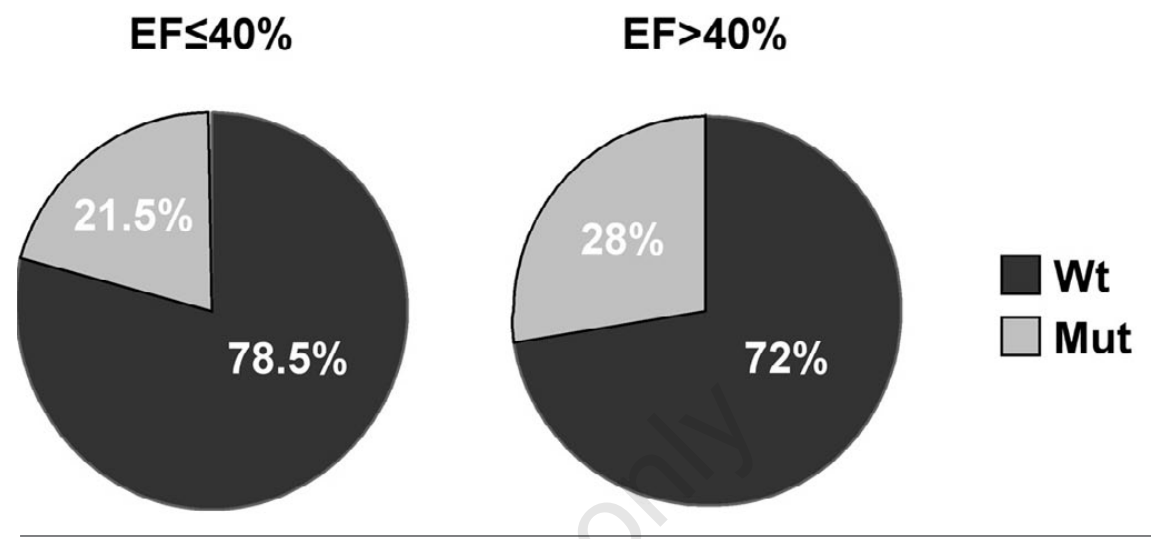

Figure 3. MMP9 polymorphism and LV remodeling after MI. Two groups of patients were selected from the population of MI patients depending on their EF: EFE $40 \%(n=79)$ and $\mathrm{EF}>40 \%(\mathrm{n}=389)$. Pie-charts represent the proportion of patients having (mut, mutated) or not (wt, wild-type) the polymorphism in each group. Differences were not statistically significant $(P=0.43)$.
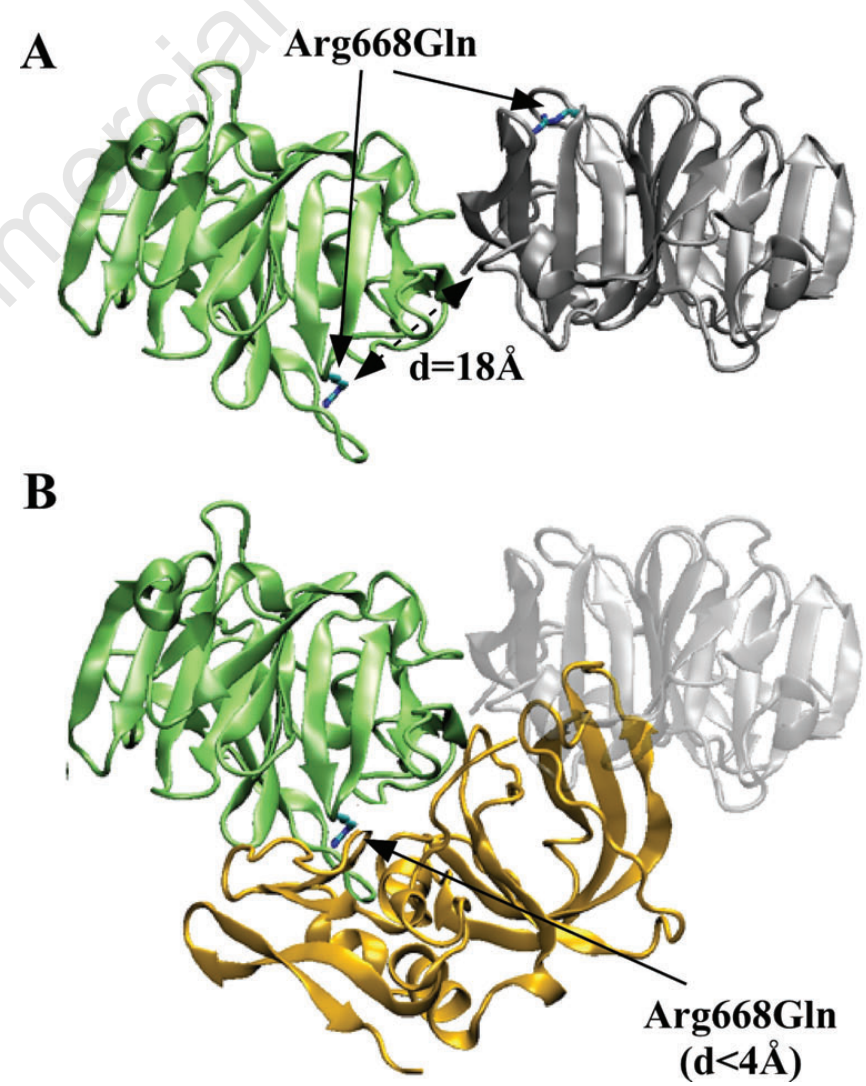

Figure 4. In silico molecular modeling of the impact of MMP9 polymorphism on PEX9/PEX9 and PEX9/TIMP1 dimers formation. The 2 PEX9 monomers are represented in green and grey, and TIMP1 monomer is in gold. Residue 668 is indicated in blue sticks. (A) The distance (d) between residue 668 of green PEX9 monomer and the closest atom of grey PEX9 monomer is $18 \AA$. (B) The distance between residue 668 of green PEX9 monomer and TIMP1 is $<4 \AA$. 
$\mathrm{P}<0.001$ (ANOVA)
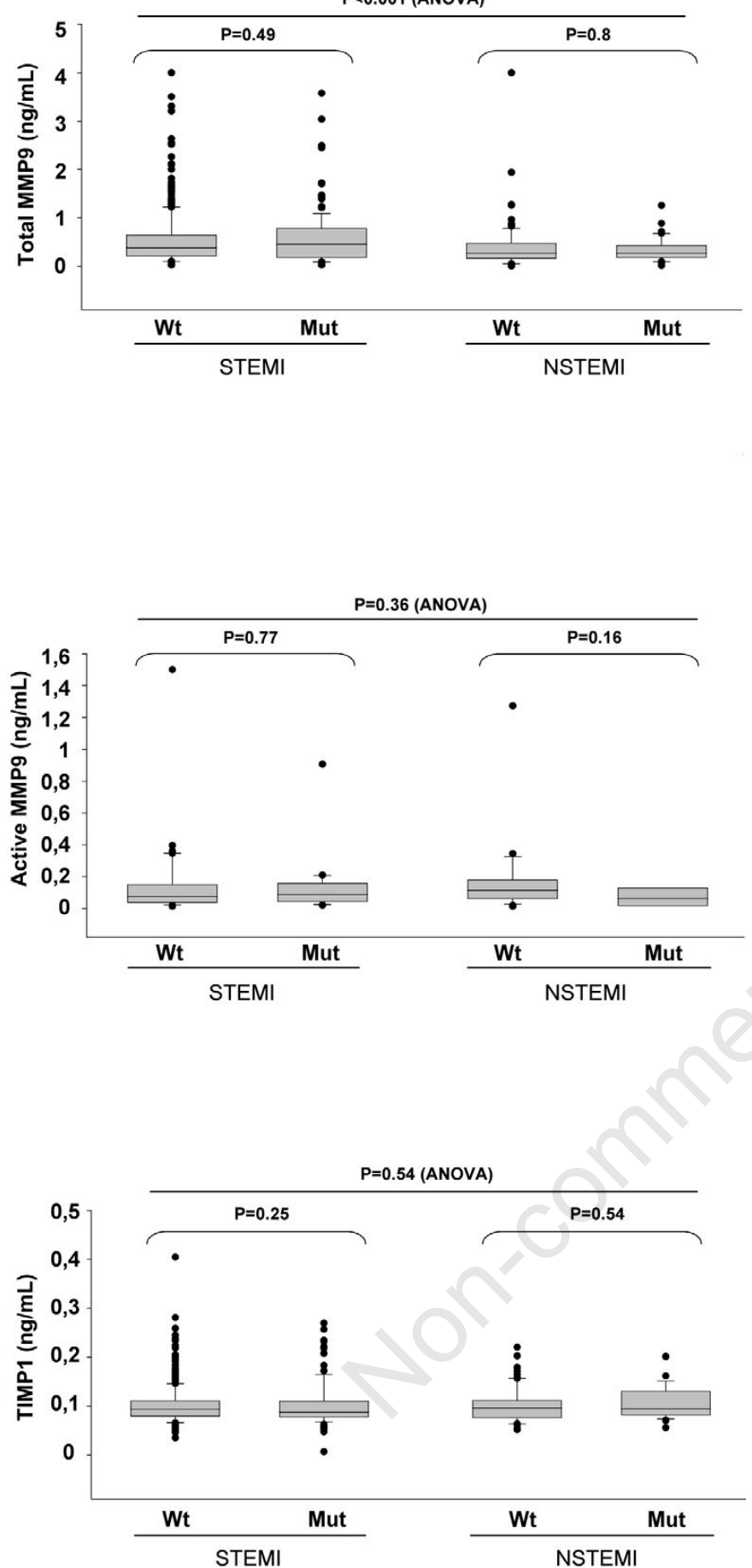

Figure 5. Association between MMP9 polymorphism and plasma levels of MMP9, active MMP9 and TIMP1. Plasma levels of total MMP9 (A), active MMP9 (B) and TIMP1 (C) were not associated with the presence of MMP9 polymorphism.
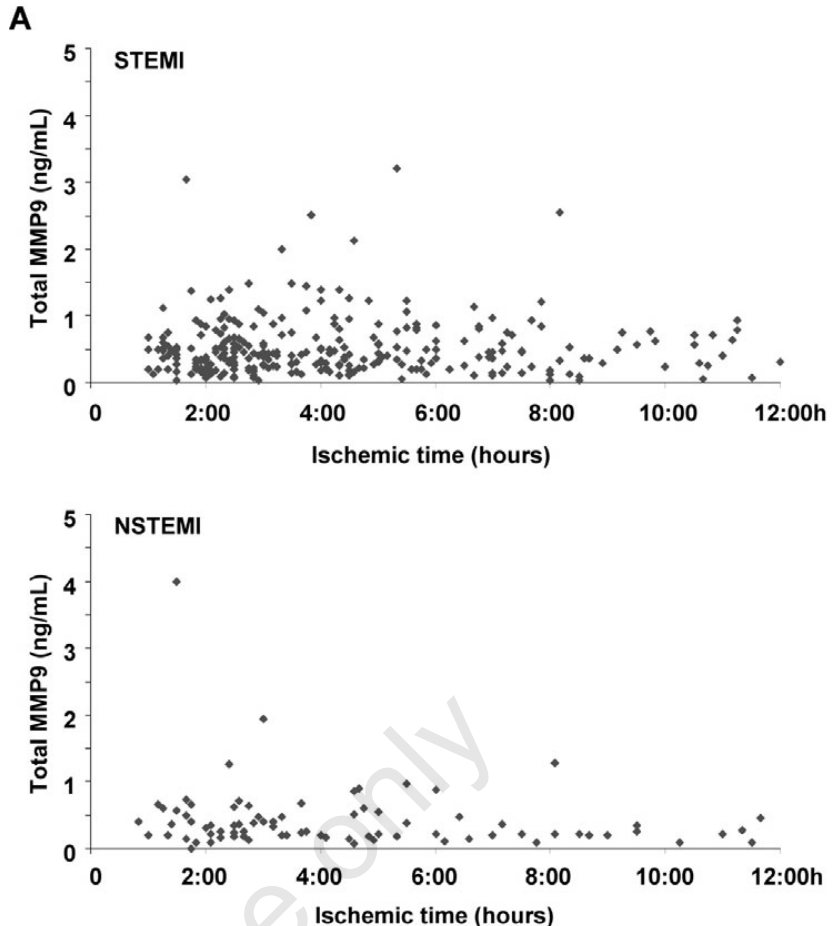

B
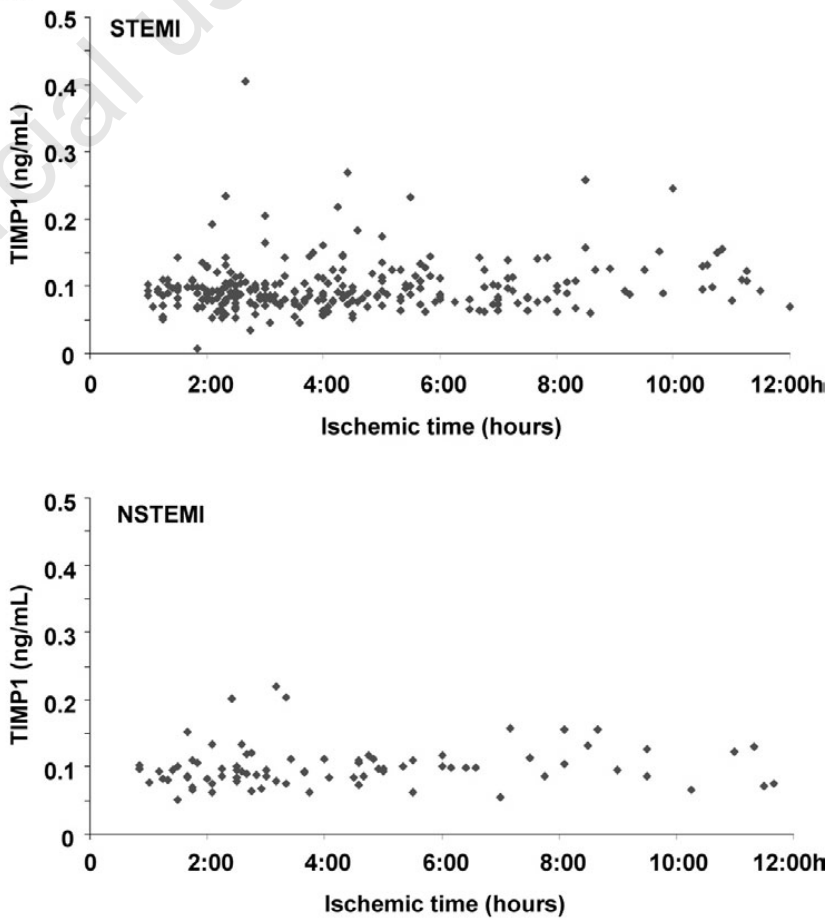

Figure 6. Plasma levels of total MMP9 and TIMP1 according to the delay between chest pain onset and blood sampling (Ischemic time) in MI patients. Total MMP9 (A) and TIMP1 (B) plasma level during the $\mathbf{1 2}$ hours following acute MI. 
more previous MI, had larger infarcts and had more HF and a higher mortality rate. Nevertheless, the MMP9 polymorphism frequency was not different between both groups. Therefore, our data suggest that the MMP9 Arg668Gln polymorphism is not associated with $\mathrm{LV}$ remodeling and dysfunction after MI.

We finally investigated whether the genetic variation could influence MMP9 activity, but we did not detect any significant variation in total and active MMP9 across the MMP9 genotypes. This is puzzling since the MMP9 Arg668Gln polymorphism is located in the coding region of MMP9, particularly in the PEX9 domain which is responsible for interactions between MMP9 and its substrates or its main inhibitor, TIMP1. One would have therefore expected that a change of the amino acid sequence of MMP9, from a basic (Arg) to a neutral residue (Gln), would have affected MMP9 activity. This was indeed suggested by in silico molecular modeling, which clearly showed the proximity of residue 668 with the interface between PEX9 and TIMP1. Eighteen additional hydrogen bounds between PEX9 and TIMP1 as well as highly different binding energies induced by the aminoacidic substitution were also in favor of an effect of the variation on MMP9 activity. From our in silico results, we had anticipated that the Arg668Gln polymorphism alters MMP9 activity. Molecular modeling suggested that the stability of the complex PEX9/TIMP1 was enhanced by the substitution, probably resulting in decreased MMP9 activity. But the PEX9 domain is also the site for binding of MMP9 substrate gelatin ${ }^{25}$ and this binding could be modified by the genetic variation as well. Moreover, TIMP1 might also play a role in proMMP9 activation, as suggested by the ability of TIMP2 to activate MMP2. ${ }^{26-28}$ It has to be acknowledged that we had only 92 active MMP9 values for MI patients, which considerably hampers the observed lack of association between active MMP9 levels and the polymorphism.

In conclusion, our report shows that the MMP9 Arg668Gln polymorphism is not associated with cardiac dysfunction and heart failure after MI.

\section{References}

1. Spinale FG. Myocardial matrix remodeling and the matrix metalloproteinases: influence on cardiac form and function. Physiol Rev 2007;87:1285-342.

2. Kai H, Ikeda H, Yasukawa H, et al. Peripheral blood levels of matrix metalloproteases- 2 and -9 are elevated in patients with acute coronary syndromes. J Am Coll Cardiol 1998;32:368-72.

3. Lindsey M, Wedin K, Brown MD, et al. Matrix- dependent mechanism of neutrophil-mediated release and activation of matrix metalloproteinase 9 in myocardial ischemia/reperfusion. Circulation 2001; 103:2181-7.

4. Squire IB, Evans J, Ng LL, et al. Plasma MMP9 and MMP-2 following acute myocardial infarction in man: correlation with echocardiographic and neurohumoral parameters of left ventricular dysfunction. J Card Fail 2004;10:328-33.

5. Sundstrom J, Evans JC, Benjamin EJ, et al. Relations of plasma matrix metalloproteinase-9 to clinical cardiovascular risk factors and echocardiographic left ventricular measures: the Framingham Heart Study. Circulation 2004;109:2850-6.

6. Webb CS, Bonnema DD, Ahmed SH, et al. Specific temporal profile of matrix metalloproteinase release occurs in patients after myocardial infarction: relation to left ventricular remodeling. Circulation 2006; 114:10207.

7. Wagner DR, Delagardelle C, Ernens I, et al. Matrix metalloproteinase-9 is a marker of heart failure after acute myocardial infarction. J Card Fail 2006;12:66-72.

8. Kelly D, Khan SQ, Thompson M, et al. Plasma tissue inhibitor of metalloproteinase-1 and matrix metalloproteinase-9: novel indicators of left ventricular remodelling and prognosis after acute myocardial infarction. Eur Heart $\mathrm{J}$ 2008;29:2116-24.

9. Kelly D, Cockerill G, Ng LL, et al. Plasma matrix metalloproteinase- 9 and left ventricular remodelling after acute myocardial infarction in man: a prospective cohort study. Eur Heart J 2007;28:711-8.

10. Yan AT, Yan RT, Spinale FG, et al. Plasma matrix metalloproteinase-9 level is correlated with left ventricular volumes and ejection fraction in patients with heart failure. J Card Fail 2006;12:514-9.

11. Matsumura S, Oue N, Nakayama H, et al. A single nucleotide polymorphism in the MMP9 promoter affects tumor progression and invasive phenotype of gastric cancer. $\mathrm{J}$ Cancer Res Clin Oncol 2005;131:19-25.

12. Hu Z, Huo X, Lu D, et al. Functional polymorphisms of matrix metalloproteinase- 9 are associated with risk of occurrence and metastasis of lung cancer. Clin Cancer Res 2005;11:5433-9.

13. Jin G, Miao R, Hu Z, et al. Putative functional polymorphisms of MMP9 predict survival of NSCLC in a Chinese population. Int $\mathrm{J}$ Cancer 2009;124:2172-8.

14. Nan H, Niu T, Hunter DJ, Han J. Missense polymorphisms in matrix metalloproteinase genes and skin cancer risk. Cancer Epidemiol Biomarkers Prev 2008;17:3551-7.

15. Koh YS, Chang K, Kim PJ, et al. A close relationship between functional polymorphism in the promoter region of matrix metalloproteinase- 9 and acute myocardial infarction.
Int J Cardiol 2008;127:430-2.

16. Hlatky MA, Ashley E, Quertermous T, et al. Matrix metalloproteinase circulating levels, genetic polymorphisms, and susceptibility to acute myocardial infarction among patients with coronary artery disease. Am Heart J 2007;154:1043-51.

17. Kaplan RC, Smith NL, Zucker S, et al. Matrix metalloproteinase-3 (MMP3) and MMP9 genes and risk of myocardial infarction, ischemic stroke, and hemorrhagic stroke. Atherosclerosis 2008;201:130-7.

18. Murphy G, Knauper V. Relating matrix metalloproteinase structure to function: why the "hemopexin" domain? Matrix Biol 1997; 15:511-8.

19. Wu S, Hsu LA, Teng MS, et al. Association of matrix metalloproteinase 9 genotypes and cardiovascular disease risk factors with serum matrix metalloproteinase 9 concentrations in Taiwanese individuals. Clin Chem Lab Med 2010;48:543-9.

20. Cha H, Kopetzki E, Huber R, et al. Structural basis of the adaptive molecular recognition by MMP9. J Mol Biol 2002;320:1065-79.

21. Gomis-Ruth FX, Maskos K, Betz M, et al. Mechanism of inhibition of the human matrix metalloproteinase stromelysin-1 by TIMP-1. Nature 1997;389:77-81.

22. Eisenstein M, Shariv I, Koren G, et al. Modeling supra-molecular helices: extension of the molecular surface recognition algorithm and application to the protein coat of the tobacco mosaic virus. $\mathrm{J}$ Mol Biol 1997;266:135-43.

23. Katchalski-Katzir E, Shariv I, Eisenstein M, et al. Molecular surface recognition: determination of geometric fit between proteins and their ligands by correlation techniques. Proc Natl Acad Sci USA 1992;89:2195-9.

24. Case DA, Darden TA, Cheatham TE, et al. Amber 8. San Francisco: University of California; 2004.

25. Roeb E, Schleinkofer K, Kernebeck T, et al. The matrix metalloproteinase 9 (mmp-9) hemopexin domain is a novel gelatin binding domain and acts as an antagonist. J Biol Chem 2002;277:50326-32.

26. Murphy G, Nagase H. Progress in matrix metalloproteinase research. Mol Aspects Med 2008;29:290-308.

27. Butler GS, Butler MJ, Atkinson SJ, et al. The TIMP2 membrane type 1 metalloproteinase "receptor" regulates the concentration and efficient activation of progelatinase A. A kinetic study. J Biol Chem 1998;273:871-80.

28. Lichte A, Kolkenbrock H, Tschesche H. The recombinant catalytic domain of membranetype matrix metalloproteinase-1 (MT1-MMP) induces activation of progelatinase $\mathrm{A}$ and progelatinase A complexed with TIMP-2. FEBS Lett 1996;397:277-82. 